\title{
The Thermocapillary Effects in Phase Change Materials in Microgravity experiment: Design, preparation and execution of a parabolic flight experiment
}

\author{
J.M. Ezquerro, A. Bello, P. Salgado Sánchez ${ }^{*}$, A. Laveron-Simavilla, V. Lapuerta
}

The Thermocapillary Effects in Phase Change Materials in Microgravity (TEPiM) experiment aims to analyse the
potential of Marangont convection for heat transfer enhancement in Phase Change Materials (PCMs) in
weightlessness, where the slow characteristic time of the phase change process constrains their use as thermal
control devices. On ground, natural convection masks the influence of Marangoni flow, which means that
quantifying each contribution to the heat transfer rate of the system precisely is not possible. In this sense, the
microgravity environment provided by parabolic flights gives an excellent opportunity to execute an experiment
on PCMs phase change coupled with thermocapillary convection, and retrieve useful data to validate theoretical
and numerical models. We note that such microgravity research has never been performed prior to this project.
ln this paper, we describe the design, preparation and performance of the TEPiM microgravity experiment,
executed during the 65th ESA Parabolic. Flight campaign. The experiment cells, filled with solid n-octadecane
and a layer of air, were heated to observe the melting process under the microgravity portions provided during
the flight. The promising results obtained support the positive impact of thermocapillary effects in PCMs
melting, and suggest themselves for further investigation. We also expect that the valuable lessons learned
presented here will help the scientific community in developing future microgravity experiments of this nature.

\section{Introduction}

During melting and solidification processes, Phase Change Materials (PCMs) naturally store and release a large amount of heat energy. The latent heat of the solid/liquid (from now on, $S / L$ ) phase transition could be exploited to maintain the temperature of a system within a sate range, with no energy consumption associated. This large thermal storage capacity and the great thermal stability shown around the melting point are two key factors for many industrial applications, where PCMs are used as passive thermal control devices. Electronic cooling, air conditioning in buildings, waste heat recovering, or compensating the time offset between energy production and consumption in solar power plants, are just a few examples of their vast applicability [1].

The thermal cycles present during the operation of devices onboard spacecrafts are suited as well to the heat storage and discharge capabilities of PCMs. Space systems have used PCMs for a wide variety of both low and high temperature applications in microgravity, ranging from complex engineering applications, like temperature peaks avoidance in electronic devices, or control of electrical power components and batteries in lunar and Mars rovers; to daily needs like refrigerating food and biological waste samples in manned missions [2].

Nowadays, hundreds of natural and synthetic PCMs with a broad range of operating temperatures are available. The most common are paraffins, fatty acids and salt hydrates. However, a major issue in thermal control with these materials, especially paraffins at the working temperatures of spacecrafts, is their low thermal conductivity. This leads to long heat storage and discharge phases, reducing their performance and usability for heat control in space.

On ground, the most extended approach to alleviate such conductivity constraint is to promote convection in the liquid phase of the system. In the presence of normal gravity, this natural (convective) motion enhances the heat transfer rate about an order of magnitude. In microgravity, conversely, the lack of natural convection requires other artificial approaches. A fairly well established solution results from placing large areas of PCM in contact with high conductive materials, 
like metallic fins or foams [3]. Whereas this solution is also applicable onboard spacecrafts, it increases the mass and size of thermal control devices and compensates the absence of convection just partially.

Prompted from the above considerations, a mechanism to enhance heat transfer on PCMs in microgravity, without increasing the system mass and volume, was envisaged by promoting the Marangoni flow induced by surface tension gradients. Under normal gravity, the isolated effect of Marangoni-driven motion is masked by natural convection and, therefore, it is not possible to distinguish experimentally the precise contribution of each one to the heat transfer rate of the system. In addition, only a handful of theoretical studies about the influence of the thermocapillary effects in the melting of a solid were found in the literature [4-7].

Aiming to understand the possibility of using the Marangoni effect as a thermal heat enhancer in microgravity, the research group has developed a numerical model that predicts the weightless behaviour of PCMs melting in the presence of air. We draw attention on the potential of the associated Marangoni effect in simple configurations. Since the validation of this model requires experimental data, the Thermocapillary Effects in Phase Change Materials in Microgravity (TEPiM) experiment has been designed. Prior to this project, microgravity experiments of PCMs coupled with thermocapillary convection had not been performed.

In order to select the microgravity platform to execute the experiment, several options were considered. Given the characteristic time of Marangoni convection in the order of tens of seconds, drop towers, with associated microgravity periods of few seconds, were not suitable for obvious reasons. Other microgravity research environments, like sounding rockets, provide longer microgravity but lack the advantage of an insitu control of the experiment by an operator. In this sense, the possibility to perform experiments in parabolic flights provided us a perfect starting point to obtain novel experimental data, and gain further experience to prepare future sounding rocket or International space Station (ISS) experiments.

The TEPiM experiment was presented to the Fly your Thesis! 2016 call for proposals, sponsored by the educational division of the European Space Agency (ESA), the ESA Education Office. It was selected, along with other three projects, to fly onboard the A310 ZERO-G aircraft from Novespace. Experiments were executed successfully in November 2016, during the 65th ESA Parabolic Flight campaign.

Throughout the three flight days of the campaign, we performed a daily set of 18 microgravity experiments. This allowed to test different parameters and repeat experiments, ensuring reproducibility and isolating spurious results due to external factors. A previous systematic study of the different constraints associated with parabolic flights, like the $g$-level cycles or the imperfect microgravity level provided, was undertaken to identify requirements on the experiment design. They pursued, among other things, to alleviate any detrimental effect in the experiment itself and its results.

In this paper, we describe the TEPiM experiment design, preparation and performance during the 65th ESA Parabolic Flight Campaign, sponsored by the Fly your Thesis! Programme. In Section 2, we define the main scientific objectives and requirements. In Section 3, a detailed description of the design solutions for the main subsystems is presented. In Section 4, we address the operational concept and experimental procedure. In Section 5 , preliminary results and relevant lessons learned are discussed. Finally, conclusions are offered in Section 6.

\section{Scientific objectives and requirements}

The main scientific objectives and requirements of the TEPIM experiment are described here, including the relevant parameters and constraints of the parabolic flight.

\subsection{Scientific objectives}

In order to understand PCMs behaviour and validate the mathematical formulation and numerical modelling, the TEPiM project proposed the design of an experiment to study PCM dynamics and heat transfer features in the presence of Marangoni convection under microgravity conditions. Whereas the melting of a solid in presence of thermocapillary effects had been subject of intense research on crystal growth $[8,9]$ or laser processing of materials [10], the focus on PCMs for terrestrial applications has diverted the attention from PCM dynamics and heat transfer capabilities in microgravity conditions.

On ground, a large number of experiments in both simple and complex geometries were devoted to follow the advance of $\mathrm{S} / \mathrm{L}$ interfaces under the dominant effect of natural convection. Besides that, several theoretical and numerical efforts were done with the aim of predicting the observed behaviour, and were validated against this seminal research (see e.g. Refs. [11-13]).

Under microgravity conditions, on the other hand, it is well known that the melting of a solid in the absence of external forces is driven solely by conductive heat transport. The presence of a liquid-air interface and the associated Marangoni convection as a heat transport mechanism, however, has not been analysed extensively. These studies were mostly theoretical and did not help validating numerical models.

The TEPLM project aims at narrowing this gap with experiments of thermocapillary effects during the melting of PCMs, further pursuing the enhancement of their performance as thermal control devices for future space missions. Therefore, the two main scientific objectives are:

1. Measure the influence of Marangoni convection in the heat transport process during the solid-liquid phase change of PCMs in microgravity, by means of the systematic execution of experiments.

2. Use the obtained experimental results to validate the theoretical formulation and numerical model for predicting the PCM phase change behaviour, in presence of thermocapillary forces and under microgravity conditions.

\subsection{Scientific requirements}

The main scientific requirements of the experiment are outlined below. These relate mainly to fluid properties, the characteristic times of the underlying phenomena and the parabolic flight itself, and the experiment (potential) sensitivity to g-level cycles and residual accelerations.

\subsubsection{Phase change material selection drivers}

The experiment requires the selection of a PCM with a phase change temperature complying with the following scientific, operational and safety requirements:

- High latent heat.

- High thermal conductivity in liquid and solid phases.

- Melting temperature above the ambient temperature in the ground laboratory $\left(25^{\circ} \mathrm{C}\right)$.

- Melting temperature low enough to be able to force Marangoni convection, using a heat source limited to a maximum temperature of $90^{\circ} \mathrm{C}$, for safety reasons.

These requirements drive the selection of n-octadecane paraffin as the test PCM. Further details are given in Sec. 3.1.

\subsubsection{Heat transport timescales and experiment phases}

The propagation of the $S / \mathrm{L}$ front along a liquid-air interface under microgravity conditions is driven by two contributions: 


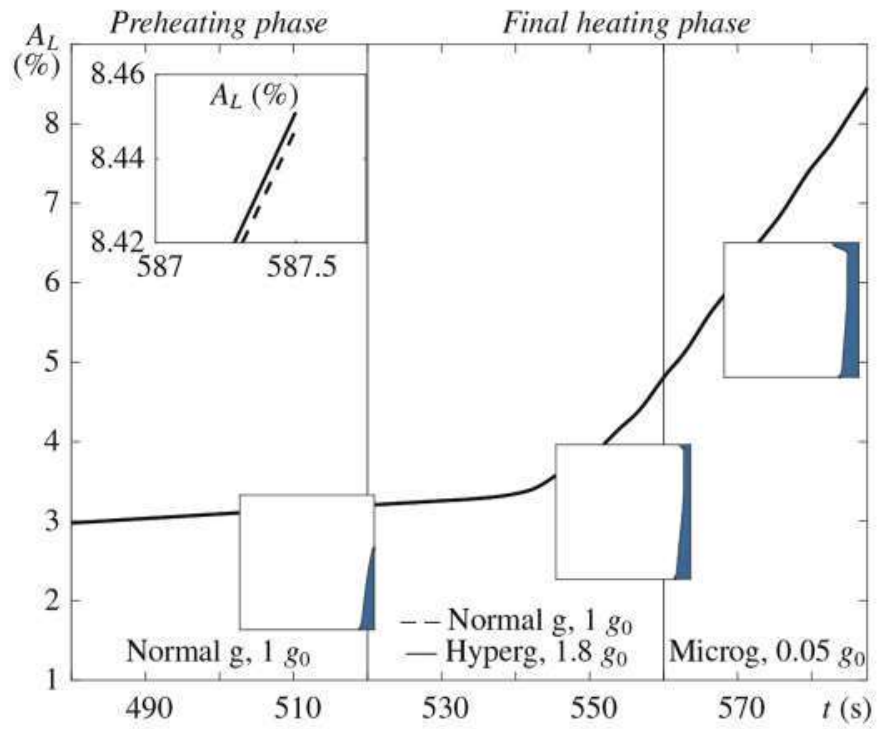

Fig. 1. Evolution of the liquid fraction (in \%, with respect the total PCM volume) of a typical TEPiM experiment with thermocapillary effect. The simulated gravity profile assumes a Preheating phase under normal gravity conditions, while the initial period of the Final heating phase is simulated under hypergravity (solid) and normal gravity (dashed line). Finally, the gravity level is reduced to $0.05 \mathrm{~g}_{0}$ for $25 \mathrm{~s}$. The coloured insets show the liquid (blue) and solid (white) phases, when hypergravity is simulated, at the end of each gravity phase. The upper-left inset illustrates a detail of the final liquid fraction in both scenarios. Simulations support a negligible impact of repeated hypergravity periods during the Preheating phase, which, assuming a cumulative and increased effect with the presence of more liquid phase, is expected to be in the order of $0.1 \%$. (For interpretation of the references to colour in this figure legend, the reader is referred to the Web version of this article.)

1. Conductive heat transport.

2. Convective heat transport driven by Marangoni convection in the melted paraffin.

For the configurations considered, a typical timescale of Marangoni convection is about $10 \mathrm{~s}$, which is orders of magnitude smaller than that of the conductive process, in the order of minutes. In fact, previous simulations showed that the Marangoni effect produced by a temperature gradient of tens of Kelvins in the liquid-air interface would be able to drive the $\mathrm{S} / \mathrm{L}$ front motion about a few millimetres (see right inset of Fig. 1, for reference) in a $25 \mathrm{~s}$ time lapse for the different geometries used. As detailed in Section 3.4, this displacement is large enough to be resolved in time and space by the experiment Optical System.

In light of the large conductive timescale, each experiment run requires a long preparation phase. This phase intends to help creating a controlled air-liquid interface at the beginning of a microgravity period and thus, to concentrate the development of Marangoni convection during the subsequent $20 \mathrm{~s}$ of microgravity, isolating its effects from convection. Finally, at the experiment completion, the test cell needs to be cooled down for safe manipulation. We anticipate that, each experiment run was divided accordingly in three phases: Preheating phase, Final heating phase and Cooling phase, corresponding to preparation, liquid-air interface creation and Marangoni convection development, and cooling steps, respectively.

\subsubsection{Airplane accelerations: microgravity level and g-level cycles}

The TEPiM experiment was carried onboard an aircraft executing parabolic manoeuvres, providing repeated periods of approximately $20 \mathrm{~s}$ of reduced gravity [14]. The microgravity level during the parabolas satisfied

$\left|g_{\mathrm{X}, \mathrm{Z}}\right| \leq 0.01 g_{0}, \quad\left|g_{\mathrm{Y}}\right| \leq 0.05 g_{0}$,

where $g_{0}=9.81 \mathrm{~m} / \mathrm{s}^{2}$; with the direction of the axes relative to the test cells as shown in Fig. 2 (a).

The balance of contact forces, surface tension and microgravity level would select the liquid paraffin location within the cell during the parabolas, generally wetting the cell interior boundaries. However, we control the liquid position via the test cell design, detailed in Sec. 3.2, that achieves the effective pinning of the contact line. This preserves a controlled liquid-air interface during the microgravity periods, almost identical to the (nearly-)flat interface observed under normal gravity conditions. The negative impact of residual gravity is therefore reduced.

These microgravity periods, on the other hand, are preceded by a pull-up phase and followed by a pull-out phase, each associated with approximately 40 and $20 \mathrm{~s}$ of hypergravity (up to $1.8 \mathrm{~g}_{0}$ ), respectively, and separated by various minutes of normal gravity $g \sim 1 g_{0}$. The potential impact of hypergravity is analysed below.

Hypergravity impact.

During the experiment execution, normal gravity periods and, specifically, hypergravity lapses drive natural convection in the liquid PCM that may affect detrimentally the relevant phenomena. A stress scenario to predict the effect of hypergravity and verify its negligible influence on the speed of the S/L front, and overall liquid fraction, was carried out numerically.

In Fig. 1, two simulations for a $20 \mathrm{~mm}$ height rectangular test cell are shown, assuming 2-dimensional behaviour (see Sec. 3.2, for

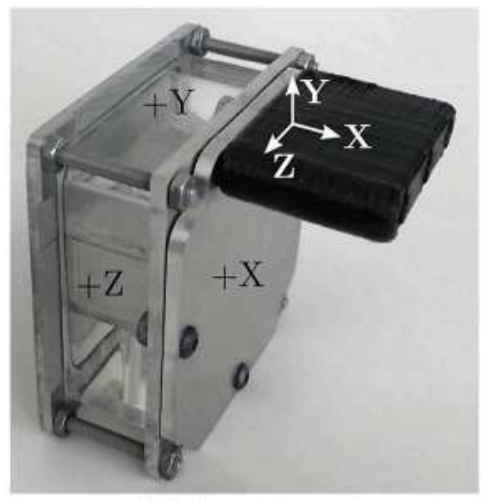

(a) General view

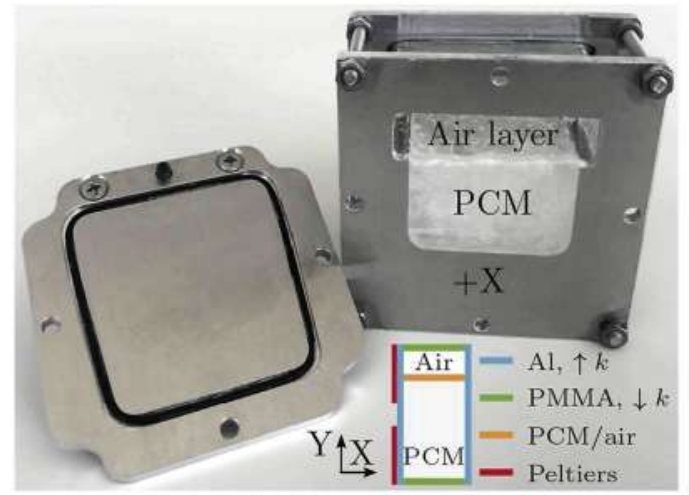

(b) Marangoni cell

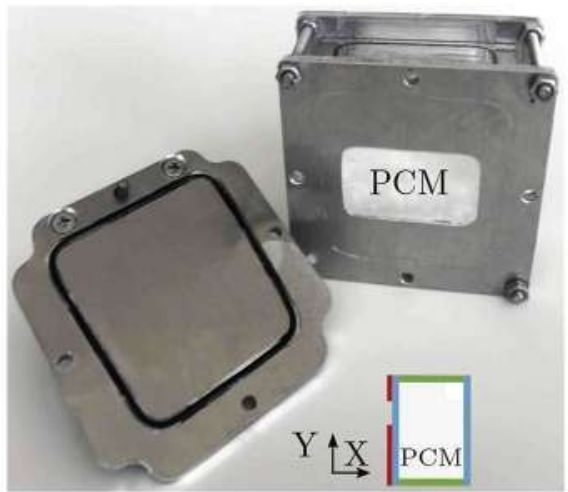

(c) Reference cell

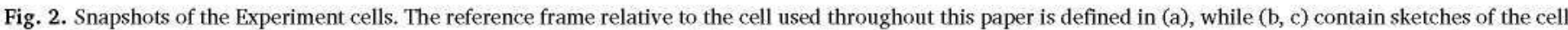

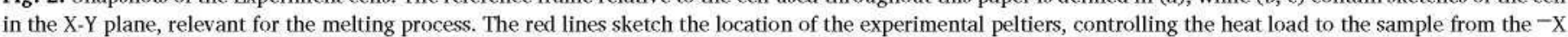

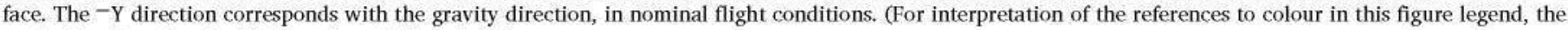
reader is referred to the Web version of this article.) 
details). The gravity level during the experiment simulation is idealised as follows:

1. Preheating phase of $8 \mathrm{~min} 50 \mathrm{~s}$ at constant temperature under normal gravity conditions, $1 \mathrm{~g}_{0}$.

2. Final heating phase period of $40 \mathrm{~s}$ under two different gravity scenarios:

(a). Hypergravity phase, where $\left|g_{\mathrm{Y}}\right|$ is increased to $1.8 \mathrm{~g}_{0}$.

(b). Extended normal gravity phase at $1 g_{0}$.

3. Final heating phase in microgravity for $25 \mathrm{~s}$, where $\left|g_{\mathrm{Y}}\right|$ is decreased to $0.05 g_{0}$.

The liquid fractions, defined as the ratio between the volume of Iiquid phase over the (total) volume of PCM, differ by less than a $0.01 \%$ in both scenarios. Therefore, simulations support a negligible impact of one hypergravity cycle.

Repeated hypergravity periods during the Preheating phase (see Sec. 4) can be analysed similarly. We may assume a cumulative effect, showing a more pronounced influence with increased liquid fractions, to estimate a total melted fraction deviation of $0.1 \%$. Consequently, a high margin of confidence on the negligible impact of the hypergravity phases is assured.

\section{Experiment design}

The experimental setup, held in two different racks, includes the following main items, explained in corresponding Sections:

1. (Sec. 3.2) Eighteen Experiment cells:

- Fifteen Marangoni cells: rectangular cuboids of three different heights (five cells each) filled with PCM and a $5 \mathrm{~mm}$ air layer on top, providing the air-liquid interface to create Marangoni convection during the melting.

- Three Reference cells: rectangular cuboids with the same three different heights (one cell each) filled with PCM. A small volume of air is left in the cell interior to act as a compensation volume.

2. (Sec. 3.3) Two Monitoring Stations, where the Experiment cells are installed to perform the experiment runs.

3. (Sec. 3.4) Two Optical Systems, one per Monitoring Station, consisting of a set of two cameras and environmental illumination. This allows for observing the PCM S/L interface during its melting from a lateral and a top view.

4. One Storage Station to stow the Experiment cells when not installed in the Monitoring Stations.

5. One Laptop and different electronic devices to control the experiment.

\subsection{Selected phase change material}

The n-octadecane paraffin displays well known and stable physical properties, detailed in Table 1 . These features make it well suited for the TEPiM experiment and permit to reuse it in different flights. The melting temperature $T_{M}$, which is its most sensitive physical property to successive melting-solidification processes, requires about 450 cycles to increase a tenth of Kelvin. The paraffin was held in cuboidal cells, described hereafter.

\subsection{Experintent cells: Marangoni and Reference cells}

The main body of the Experiment cells is a rectangular cuboid to ease their manufacture and facilitate the visualization of the $\mathrm{S} / \mathrm{L}$ and liquid-air interfaces during experiments.

Fig. 2 includes three different snapshots of the cells showing (a) a general view of an Experiment cell and $(b, c)$ two detailed views of a Marangoni cell (b), containing the volume of PCM and a $5 \mathrm{~mm}$ air layer, and the associated Reference cell (c). The cell-fixed reference frame used
Table 1

Physical properties of n-octadecane paraffin (reproduced from Refs. [15-18]).

\begin{tabular}{ll}
\hline Property & Value \\
\hline Melting temperature, $T_{M}$ & $28^{\circ} \mathrm{C}$ \\
Liquid density, $\rho_{L}$ & $780 \mathrm{~kg} / \mathrm{m}^{3}$ \\
Solid density, $\rho_{S}$ & $865 \mathrm{~kg} / \mathrm{m}^{3}$ \\
Latent heat, $L$ & $243.5 \mathrm{~kJ} / \mathrm{kg}$ \\
Liquid specific heat, $c_{p L}$ & $2196 \mathrm{~J} /(\mathrm{kg} \mathrm{K})$ \\
Solid specific heat, $c_{p S}$ & $1934 \mathrm{~J} /(\mathrm{kg} \mathrm{K})$ \\
Liquid conductivity, $k_{I}$ & $0.148 \mathrm{~W} /(\mathrm{m} \mathrm{K})$ \\
Solid conductivity, $k_{S}$ & $0.358 \mathrm{~W} /(\mathrm{m} \mathrm{K})$ \\
Thermal expansion, $\beta$ & $9.1 \cdot 10^{-4} 1 / \mathrm{K}$ \\
Dynamic viscosity, $\mu$ & $3.5406-10^{-3} \mathrm{~Pa} \mathrm{~s}$ \\
doidT & $-8.44 \cdot 10^{-5} \mathrm{~N} /(\mathrm{m} \mathrm{K})$ \\
\hline
\end{tabular}

throughout this paper is defined in panel (a), while panels (b, c) contain $X$-Y plane drawings of the cells, relevant for the melting process. These include the experiment peltiers, sketched with red lines, controlling the heat load to the sample from the $-\mathrm{X}$ face. The $-\mathrm{Y}$ direction corresponds with the gravity direction in nominal flight conditions. We note the 3 -d printed polylactic acid (PLA) handle fixed to the $+\mathrm{X}$ cell cover for safe manipulation.

The body of the cells is made by polymethyl methacrylate (PMMA) due to its suitable optical properties, along with its good material compatibility, high temperature envelop, easy mechanisation and low heat conduction. The main body is mechanised by milling a PMMA solid piece to form a structure of four faces $\left( \pm Y_{,} \pm Z\right)$, two of them represented in panels $(b, c)$ by green lines, while the other two faces $( \pm X)$ are aluminium covers (blue lines). The PMMA body and metal covers are fixed together by a four bolts mechanical union that encloses one $\mathrm{O}$ ring in each of the two leakage paths, providing the first level of containment required.

While the $-\mathrm{X}$ face cover is always installed to close this cell face, two different covers are designed to be attached to the $+\mathrm{X}$ face: closing cover, to fully close the cell; and auxiliary cover, to permit the cell filling and draining between flights. The auxiliary cover has a small opening in its upper part that allows the cleaning of the cell and the flattening of the solid-air surface after the filling (and subsequent solidification) process.

During experiments, the PCM is subjected to controlled thermal loads through the $-\mathrm{X}$ face of the cell. Since the conductivity of the PMMA is much lower than that of the aluminium, i.e.,

$\frac{k_{\mathrm{PMMA}}}{k_{\mathrm{Al}}} \approx \frac{0.18 \mathrm{~W} /(\mathrm{m} \mathrm{K})}{220 \mathrm{~W} /(\mathrm{m} \mathrm{K})} \approx 8.2 \cdot 10^{-4} \approx 1_{\text {. }}$

the heat flux through the PMMA main body can be neglected compared to the heat flux through the $-\mathrm{X}$ aluminium cover. This effect is somewhat enhanced by the 0 -rings placed in between.

Similarly, since the solid PCM conductivity is higher but similar to the PMMA $k_{S, P C M}>k_{\mathrm{PMMA}}$, it effectively acts as a thermal insulation for the liquid phase. This allows to further neglect the corresponding heat flux through the $+\mathrm{X}$ face at leading order. In this ideal scenario, all interior boundaries but the $-\mathrm{X}$ face can be modelled as adiabatic, which means that thermocapillary forces generate a one directional motion of the liquefied paraffin, reducing the complexity to a 2-dimensional problem.

In order to define the cell interior dimensions, several ground tests were performed. Inspired by the seminal experimental work of Gau and Viskanta [11] in PCMs melting, we select a cell dimension in the Z-axis of $35 \mathrm{~mm}$, which was demonstrated there to be enough to recreate a 2dimensional flow in the central plane of the cell. It is worth to point out that different behaviour is not foreseen in microgravity, since this 2dimensional nature does not depend on the particular gravity level but on viscous and local heat transport properties near the system boundaries. 


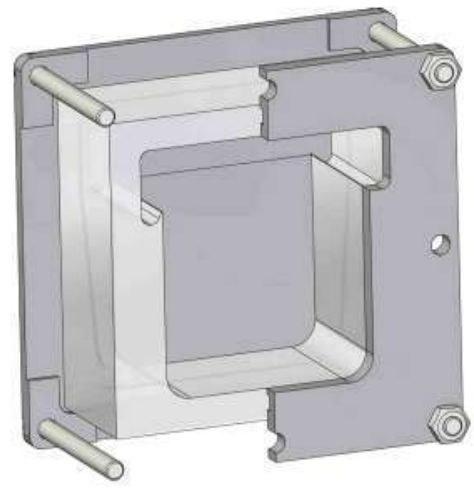

Fig. 3. CAD view of a Marangoni cell. The internal rails mechanised in the $\pm \mathrm{Z}$ PMMA faces and the $-X$ aluminium face to pin the liquid contact line can be seen in the view. A midsection cut of the aluminium cover installed in the $+X$ face is done for convenience, (For interpretation of the references to colour in this figure legend, the reader is referred to the Web version of this article.)

The cell length (in the $\mathrm{X}$-axis direction), where the $\mathrm{S} / \mathrm{L}$ interface advances, is set to $20 \mathrm{~mm}$. This selection allows the melting evolution without interfering with the opposite wall. Finally, the cell height (in the $\mathrm{Y}$-axis direction) was chosen as an experiment parameter. Cells with three different PCM heights of 20,25 and $30 \mathrm{~mm}$ values, not including the fixed air layer height of $5 \mathrm{~mm}$, were tested in order to observe their influence on the forced Marangoni convection.

Ground experiments, on the other hand, showed that the S/L interface visualization was not hindered by the different melting profiles in $\mathrm{Z}$ planes (along the cell width), caused by the (small) heat transport through the PMMA $\pm \mathrm{Z}$ walls. Near the walls, the melting evolution was slightly faster than in the centre of the cell since $k_{L, P C M}<k_{\mathrm{PMMA}}$. This, combined with the different transparency of the liquid and solid phases, permits the proper observation of the central S/L front. For a better understanding of the achieved visualization, refer to Sec. 4.

As commented above, one of the key parameters of the Marangoni cell design was the rails mechanised at the PCM/air interface, visible in Figs, 2(b) and 3, where a detailed $C A D$ view is shown. Rails were intended to pin the contact line and avoid the wetting of the air zone of the cell during the microgravity phase of the experiment. They were manufactured to provide a very sharp edge on the PMMA $\pm \mathrm{Z}$ interior faces and the $-\mathrm{X}$ aluminium cover, in contact with the liquid-air interface. Furthermore, a phobic coating ( $3 \mathrm{M}$ Novec 1702 ) was applied to improve the rail performance. Each Marangoni cell, containing PCM and air, was replicated five times to systematically repeat experiments.

Reference cells, on the other hand, were also analysed to have a reference melting process without thermocapillary effects. Three cells of 20,25 and $30 \mathrm{~mm}$ heights were filled with n-octadecane. As commented above, a small compensation volume of air inside near the $+\mathrm{X}$ face was left to ensure that the change of PCM volume did not cause any damage to the structure during experiments.

Therefore, eighteen cells were tested in each parabolic flight: fifteen Marangoni cells (five of each height) and three Reference cells. We note the substantial difference between them is the absence of the $5 \mathrm{~mm}$ air layer, needed to force Marangoni convection. Both Marangoni and Reference cells were subjected to similar thermal phases and the evolution of the S/L interface was monitored identically. To perform the experiments, each cell was installed and processed in a (so-called) Monitoring Station.

\subsection{Monitoring Station}

The Monitoring Station is where the Experiment cells are installed to perform each experimental run. For executing two runs simultaneously, two Monitoring Stations are used in parallel and placed inside a Zarges Box, which provides an additional level of containment.

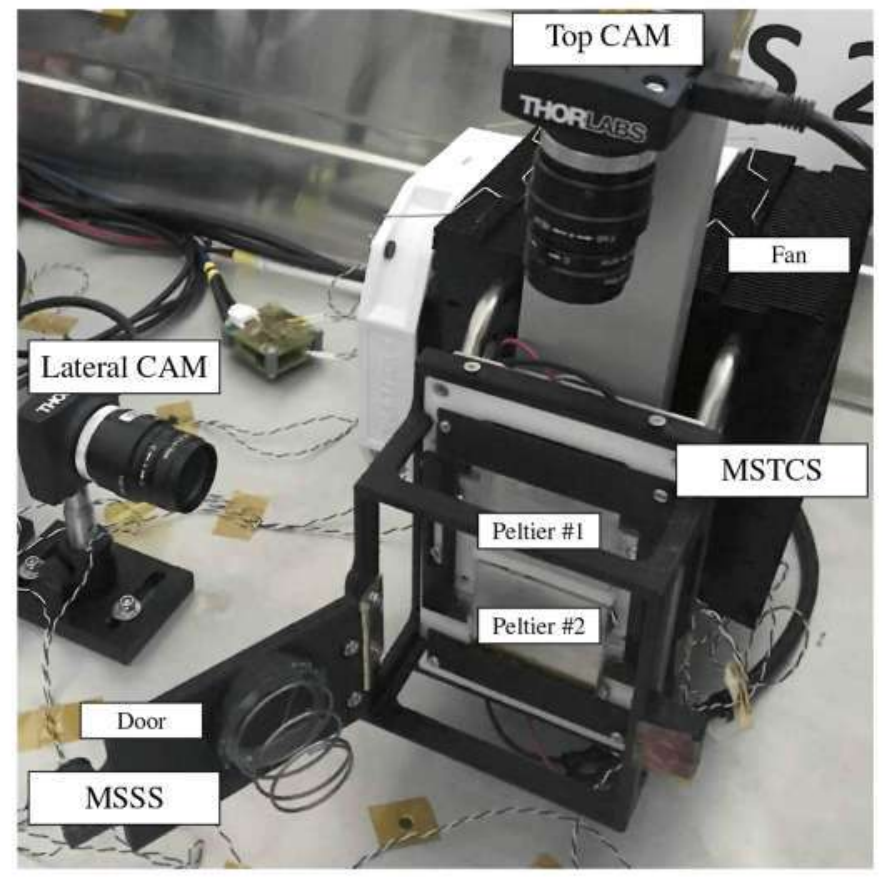

Fig. 4. General view of Monitoring Station \#2, with no Experiment cell installed. The main elements of the assembly, described in Section 3.3, are labelled for clarity. (For interpretation of the references to colour in this figure legend, the reader is referred to the Web version of this article.)

Each Monitoring Station is formed by the Monitoring Station Support System (MSSS) and the Monitoring Station Thermal Control System (MSTCS). In Fig. 4, a general view of one Monitoring Station (MS \#2) with no experimental cell installed is shown, including the locations of the lateral and top view cameras, visible in the image.

\subsubsection{Monitoring Station Support System (MSSS)}

The MSSS includes a Teflon machined piece, reinforced with an aluminium profile to support the heat exchangers; and a 3-d printed PLA structure, with a door holding a spring to support the Experiment cells. Once the door is closed, kept by two neodymium magnets, the spring presses the cell against the two heat exchangers covering the two peltiers used. This MSSS design permits to hold the cells during experiments, providing an easy and safe cells manipulation, and further maintains the best possible thermal contact between the Experiment cells - $\mathrm{X}$ face and the MSTCS.

\subsubsection{Monitoring Station Thermal Control System (MSTCS)}

The MSTCS controls the temperature of the Experiment cells $-\mathrm{X}$ face. This face is subjected to different thermal loads in its upper and lower areas, requiring the use of two peltiers, two heat exchangers, two heatsinks and one fan. Each peltier is connected to a heat exchanger, which transfers (extracts) heat to (from) the $-\mathrm{X}$ cell face, and to a heatsink. Detailed views of the MSTSC are shown in Fig. 5.

Each peltier is controlled independently to follow different temperature profiles during the experiment execution. We describe below the Active Thermal Control System implemented and the associated nominal temperature profiles.

Active Thermal Control.

An Active Thermal Control has been implemented for controlling the peltier temperatures. It is composed by one Arduino Uno, two Pololus simple motor controllers and two thermocouples. The details and data flow of the control loop are as follows:

- The thermocouples are located inside a drill performed up to the middle section of the heat exchangers. They provide temperature 


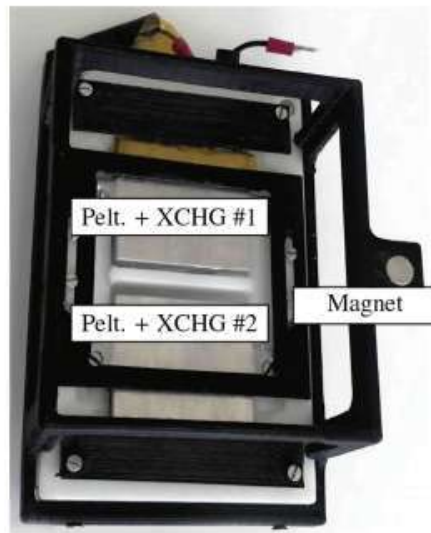

(a) MSTCS

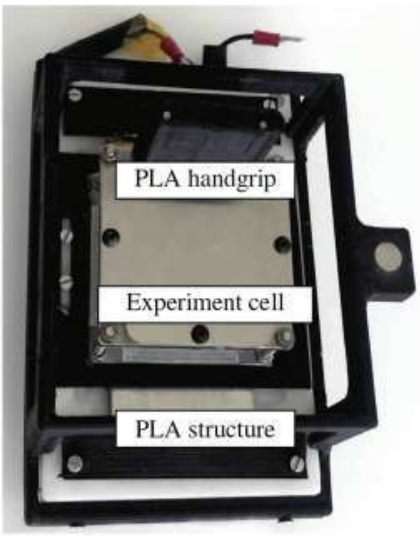

(b) MSTCS + Experiment cell
Fig. 5. Detailed views of the Monitoring Station Thermal Control System. (For interpretation of the references to colour in this figure legend, the reader is referred to the Web version of this article.)

inputs to the Arduino for the control loop.

- The Arduino receives the thermocouple signals. It calculates the power required by the peltiers to achieve the desired temperature setting, and commands the Pololu controllers. Depending on the experiment phase, it implements classical PID control or commands the saturation of the input current feeding the peltiers to reach the setpoint as fast as possible.

- The Pololu controllers are PWM capable H-Bridge Mosfet drivers that receive the information from the Arduino and control the input power coming from the power supply to the peltiers.

The experiment operators manually command the start and end of each experiment phase through a Laptop interfacing the Active Control System. The Laptop further collects and stores all data received form the Arduino.

The architecture of the Active Control System is schematised in Fig. 6, showing all relevant items and other safety devices required by Novespace. The power lines, controlled by the Pololus devices, were protected (twice) from overcurrents by the Power Supply (TENMA $30 \mathrm{~V} / 5 \mathrm{~A}$ dual channel DC power supply) integrated protection system and one extra fuse in each line. In addition, one thermoswitch and one thermofuse were placed in each power line to shut off the experiment if temperatures exceeded the permitted limits.

Peltier temperature profiles for experiments.

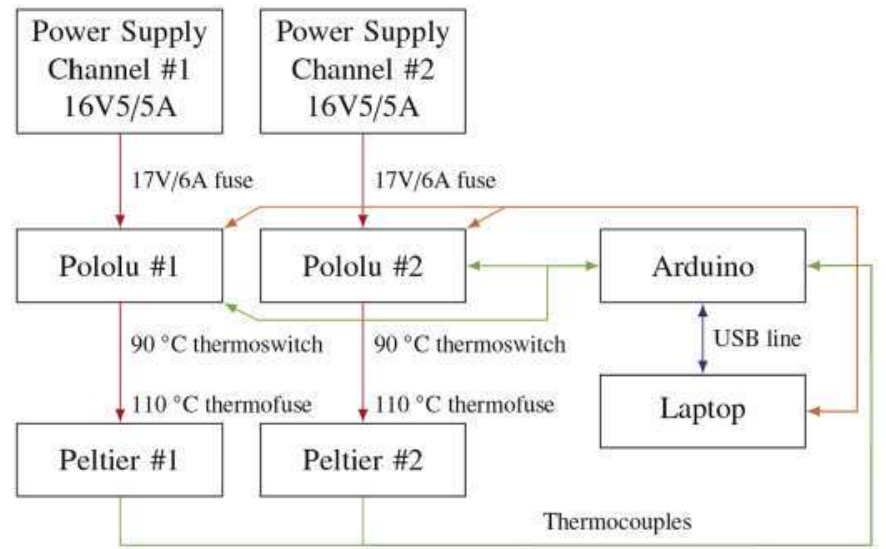

Fig. 6. Active Thermal Control schematic view. Each line type is referred in different colour: power lines (red), data lines (green), not compulsory data lines (orange), USB line (blue, power and data). (For interpretation of the references to colour in this figure legend, the reader is referred to the Web version of this article.)
During the Preheating phase (beginning) of experiments, starting roughly $8 \mathrm{~min}$ prior to the relevant microgravity phase, peltier \#1 is set to a temperature below the n-octadecane melting point $T_{1} \approx 0^{\circ} \mathrm{C}$. This peltier controls the upper side temperature of the cell $-\mathrm{X}$ face. The lower side, subjected to peltier \#2 is set to a thermal setting well over the melting temperature $T_{2} \approx 80^{\circ} \mathrm{C}$.

Around $1 \mathrm{~min}$ before the afore-mentioned microgravity period, and lasting until the relevant parabola completion, the Final heating phase starts and both peltier temperatures are controlled at the same setting $T_{1} \approx T_{2} \approx 80^{\circ} \mathrm{C}$. For further details, see Fig. 9 , where temperature profiles of an experiment are illustrated.

The control provided by peltier \#1 is achieved by placing it between a big heatsink, in combination with a fan and a heat exchanger (aluminium cover piece). During the Preheating phase, the peltier transfers a great amount of heat from the cell to the heatsink. As the temperature of the PCM shall be maintained below $28^{\circ} \mathrm{C}$, a big heatsink is required to evacuate the heat to the ambient air. Therefore, the heatsink temperature remains low and the peltier is able to maintain a controlled temperature difference between its sides, providing a $T_{1} \approx 0^{\circ} \mathrm{C}<T_{M}$. In addition, at the experiment completion, peltier \#1 temperature is reduced from roughly 80 to $28^{\circ} \mathrm{C}$ to cool down the cell, step in which the heatsink-fan assembly helps evacuating a great amount of heat in a short period of time.

The control provided by peltier \#2, on the other hand, is achieved by placing peltier \#2 between a small heatsink (in comparison with the one used on peltier \#1) and a heat exchanger. Since the thermal performance of peltier \#2 is less demanding for the system, the use of a fan is not required to achieve (and maintain) the target temperatures.

\subsection{Optical System}

The camera selected for the TEPiM experiment is the ThorLabs DCC $1545 \mathrm{~m}$, a CMOS camera that has a maximum frame rate of 25 FPS at the maximum resolution of $1280 \times 1024$ pixels. The MVL8M23 camera lenses from ThorLabs are used with the camera. Camera and lens pair with the same format $\left(2 / 3^{\prime \prime}\right)$ to avoid cropping and vignetting of the images and to have the camera field of view (FOV) at the maximum allowed by the lens. The lens has a depth of field (DOF) of around $45 \mathrm{~mm}$, which allows the interior of the experimental cells to be observed. A summary of the main camera and lens characteristics is given in Table 2.

Two cameras, aligned with the $\mathrm{Y}$ and $\mathrm{Z}$ axes of the Experiment cells, record the evolution of the $\mathrm{S} / \mathrm{L}$ interface. They are powered and controlled via USB connection with the Laptop, where the acquired videos are stored. We select different acquisition rates according to each experiment phase. In this sense, Preheating and Cooling phases were monitored at approximately $1 \mathrm{~Hz}$, while an increased rate of $10 \mathrm{~Hz}$ was used during the Final heating phase, when the Marangoni convection occurs.

A commercial LED array is used for illumination. The model EGLO LED DECO is a $3 \mathrm{~m}$ LED strip which includes a $12 \mathrm{~V}$ power supply and has a maximum power consumption of $13.5 \mathrm{~W}$ with $250 \mathrm{~lm}$.

Table 2

Camera, lens and sensor characteristics.

\begin{tabular}{lll}
\hline & Lateral view & Upper view \\
\hline Format & $2 / 3^{\prime \prime}$ & $2 / 3^{\prime \prime}$ \\
FOV & $67^{\circ}$ & $67^{\circ}$ \\
Min. object distance & $120 \mathrm{~mm}$ & $120 \mathrm{~mm}$ \\
Focal length & $8^{\prime \prime}$ & $8^{\prime \prime}$ \\
Horiz. resol. (pixel/mm) & 12.9 & 11.5 \\
Vert. resol $\left(\mathrm{pixel} / \mathrm{mm}^{\prime}\right)$ & 12.4 & 13.7 \\
Area resol. $\left(\mathrm{pp}^{2} / \mathrm{mm}^{2}\right)$ & 160.0 & 157.5 \\
\hline
\end{tabular}


3.5. Onboard configuration: electronic-monitoring rack and housing box rack

The Laptop, electrical system and Active Thermal Control were located on Rack \#1. Rack \#1 wt $45 \mathrm{~kg}$ and its volume is approximately $300 \mathrm{~L}$. The power supplied by the aircraft $(220 / 240 \mathrm{~V}$ alternate voltage $)$ feeds the following elements:

- Laptop.

- Two laboratory power supplies to feed the four peltiers $(16.5 \mathrm{~V}, 5 \mathrm{~A}$ maximum for each peltier).

- The voltage converter (12 V) that feeds the fans and the LED string $(1.25 \mathrm{~A})$.

The Monitoring Stations and Optical Systems, on the other hand, were placed inside a Zarges box that provides the required second level of containment to test fluids. To ensure the leakproofness of the box, cables are passed through sealed cable glands. Taking into account the possibility of cabin depressurisation, the Housing Box includes a coil made of flexible hose of $4 \mathrm{~mm}$ inner diameter, and a rupture device that releases the air when breaking. The Housing Box was mounted on Rack \#2 with a total weight (including the fixation bars) of $50 \mathrm{~kg}$ and a volume of $259 \mathrm{~L}$.

Finally, the Storage Station, which was used to stow the Experiment cells during the flights, was fixed directly to the cabin rails and its weight and volume were approximately $3 \mathrm{~kg}$ and $15 \mathrm{~L}$.

These provided a total approximate weight and volume of $100 \mathrm{~kg}$ and $575 \mathrm{~L}$, respectively.

\section{Operational concept: experiment procedure and scientific data}

To comply with the timescales explained in Section 2.2.2, each experiment run is divided in the three different phases as follows.

Preheating phase. The afore-mentioned objective of this phase is to help creating the air-liquid interface in a short characteristic time $(\sim$ $20 \mathrm{~s}$ of microgravity). For this purpose, we drive the formation of a liquid paraffin bubble in the lower side of the cell, surrounded by solid octadecane [see Fig. 7(b)].

This approach prevents the liquid confined by the solid paraffin to escape and wet the cell zone filled with air due to the $g$-cycles during the flight. Upon Preheating phase completion, enough liquid shall be present to facilitate the fast melting of the upper solid part, which is subjected to the upper peltier.

During the Preheating phase, peltier temperatures are set to two different values:

1. Peltier \#1 temperature $T_{1} \approx 0{ }^{\circ} \mathrm{C}$, well below the octadecane melting temperature.

2. Peltier \#2 temperature $T_{2} \approx 80^{\circ} \mathrm{C}$, below $90^{\circ} \mathrm{C}$ (maximum temperature allowed due to flight safety) and well above the octadecane melting temperature.

The real duration of this phase, nominally $8 \mathrm{~min}$, varied somewhat between 7 and $11 \mathrm{~min}$, according to the real flight execution.

Final heating phase. During this phase, peltier temperatures are set to $T_{1} \approx T_{2} \approx 80^{\circ} \mathrm{C}$ around $40 \mathrm{~s}$ before the microgravity phase starts. The solid layer (solid bridge) separating the liquid paraffin from the air is melted [see Fig. 7(c)], creating the liquid-PCM/air interface after, roughly, half a minute.

The convection created by thermocapillary effects in the octadecane liquid increases the heat transmission, accelerating the melting and the motion of the S/L interface at the liquid-air interface [see Fig. 7(d)].

Cooling phase. At the experiment end, once the microgravity phase is completed, the cell is cooled down for its safe removal, and the Monitoring Station is prepared for the following experiment run.

Provided the experiment durations were several minutes, it was not

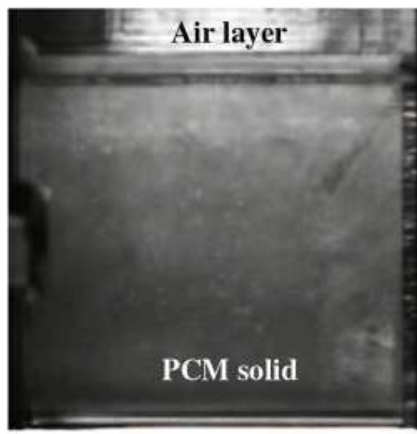

(a) Preheating phase start: $t=0 \mathrm{~s}$

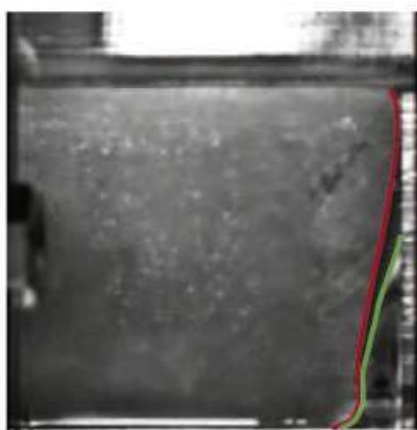

(c) Final heating phase: $t=551 \mathrm{~s}$

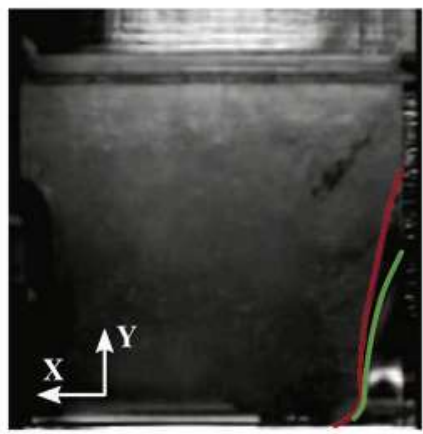

(b) Preheating phase end: $t=533 \mathrm{~s}$

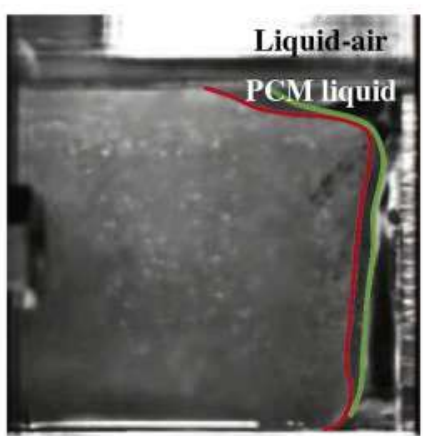

(d) Final heating phase end: $t=612 \mathrm{~s}$

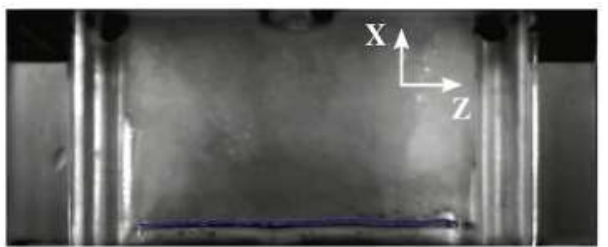

(e) Upper view, Final heating phase end: $t=612 \mathrm{~s}$

Fig. 7. (Colour online) Snapshots showing the temporal evolution of an experiment using a Marangoni cell: (a-d) lateral and (e) top views, (a) Preheating phase, $t=0 \mathrm{~s}$. (b) End of the Preheating phase, lasting around $9 \mathrm{~min}$, with the formation of the liquid bubble. (c) After 20 s of the Final heating phase, showing the melting of the upper solid part. (d, e) End of the experiment, showing the faster melting in the air-liquid interface due to Marangoni convection. For visual reference, the (approximate) S/L front in the lateral wall (red) and central plane (green) are highlighted. (For interpretation of the references to colour in this figure legend, the reader is referred to the Web version of this article.)

possible to perform runs in every parabola. To maximise the scientific output of the campaign, two Monitoring Stations were used in parallel and a detailed plan of the flight was developed. The runs in each Monitoring Station were scheduled in different parabolas to obtain results more statistically representative.

We describe below the experiment sequence executed in each flight and the experiment parameters varied between runs. Finally, the experiment data collected is reviewed.

\subsection{Experiments sequence}

Fig. 8 shows the experiments sequence for the parabola groups 1 and 2 in the first flight. The subsequent timeline followed a similar script, configuring the daily operations sequence for each flight.

Following this detailed flight plan, three parabolas per group of five were meaningful for observing the relevant phenomena, conducting a daily set of 18 experiments in different test cells: five Marangoni cells of each height (20, 25 and $30 \mathrm{~mm}$ PCM heights), and their corresponding Reference cells. In all the flights, we used parabola 0 for the experiment operators adaptation to the changing gravity environment. 


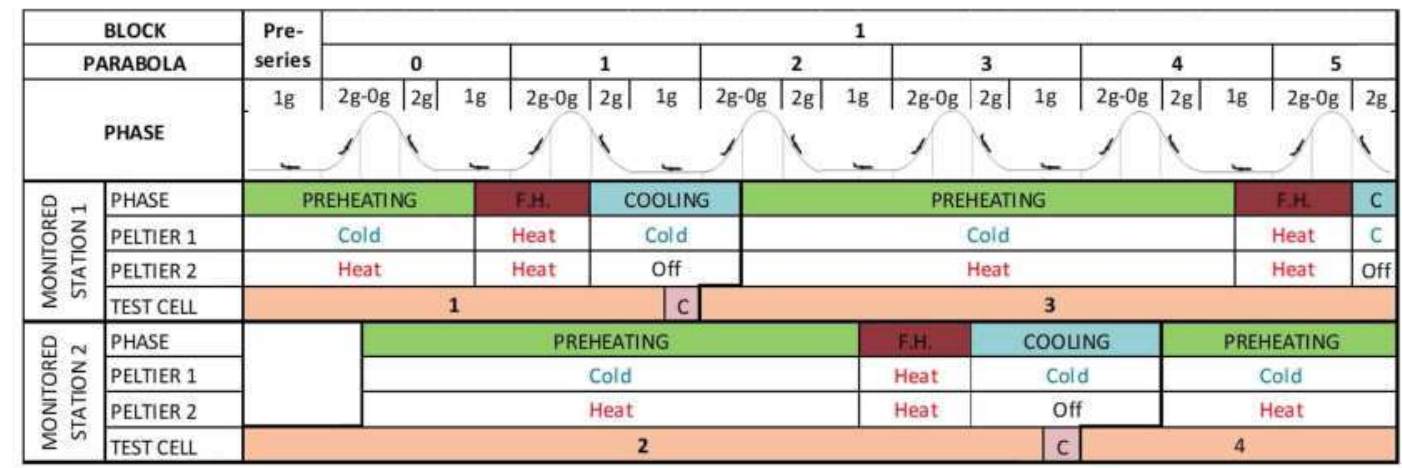

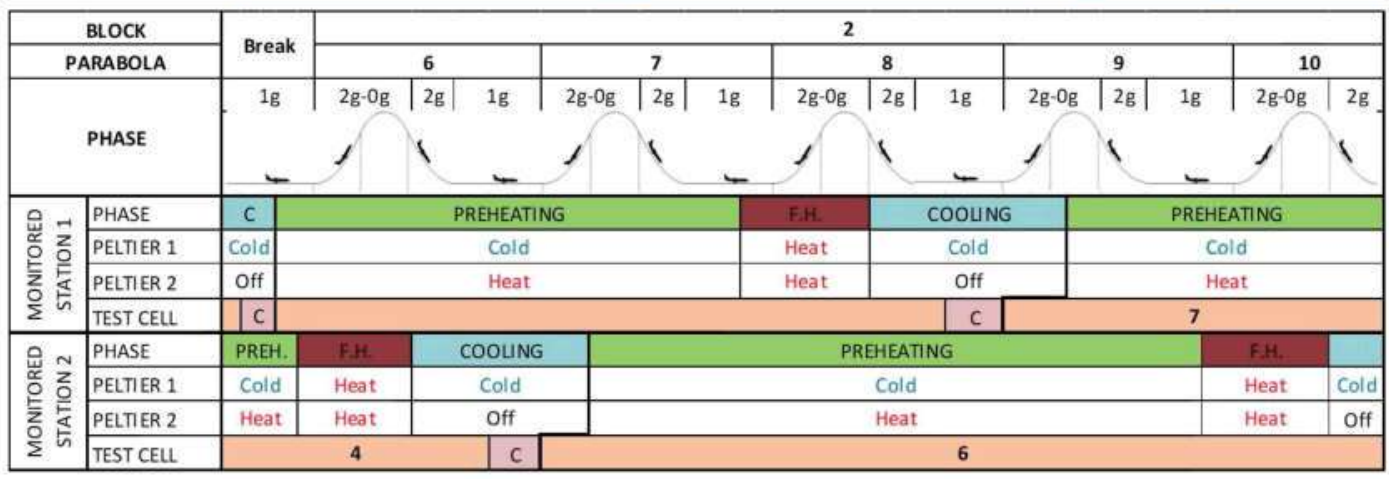

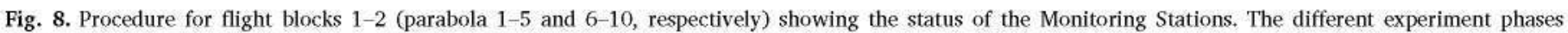
(Preheating phase, Final heating phase and Cooling phase), peltier states, processed cell and other operationally relevant parameters are detailed.

Table 3

Range of experiment parameters in each flight. Note that despite a nominal Preheating phase duration of $8 \mathrm{~min}$ was foreseen, the real duration was determined by the actual flight execution and, therefore, not controlled by the experiment operators.

\begin{tabular}{llll}
\hline Flight & 1st & 2nd & 3rd \\
\hline$T_{1}$ - Preheating (C) & $-5,0$ & $-5,0$ & $-5,0$ \\
$T_{1}$ - Final heating (C) & $80,82.5$ & $80,82.5$ & 82.5 \\
$T_{2}-$ Preheating (C) & $65,72.5$ & 65,70 & $62.5,70$ \\
$T_{2}$ - Final heating (C) & $75,77.5$ & $77.5,80$ & $77.5,80$ \\
Preheating duration (s) & 350,560 & 430,710 & 410,680 \\
\hline
\end{tabular}

Two operators were required for the TEPiM experiment execution. During the flight, operator \#1 was responsible of Rack \#1, performing control and monitoring tasks via the Laptop, while operator \#2 was in charge of the installation and removal of the Experiment cells in the Monitoring Stations, focusing on tasks related to Rack \#2.

For the removal of an Experiment cell, the Zarges box was opened and the corresponding Monitoring Station set to $26^{\circ} \mathrm{C}$. After waiting an approximate cool down time of $20 \mathrm{~s}$, operator \#2 wearing thermal protective gloves opened the Monitoring Station Support System door and, holding the Experiment cell by its PLA handgrip, moved it to a temporal stow location. Then, operator \#2 grabbed a fresh Experiment cell from the Storage Station and installed it in the Monitoring Station Support System. Finally, the processed Experiment cell was stowed into the Storage Station and the Zarges box was closed in preparation for the next parabola.

\subsection{Experiment parameters}

The experiment parameters are:

1. Experiment cell height (volume). As mentioned in Section 3.2, three different PCM heights of 20,25 and $30 \mathrm{~mm}$ were tested for both Marangoni and Reference cells.
2. Peltier temperatures $T_{1}$ and $T_{2}$. These parameters modify the shape of the liquid bubble generated during the Preheating phase and, therefore, modifies the position of the $\mathrm{S} / \mathrm{L}$ interface and its velocity during the Final heating phase. Different temperature values were tested in order to study the influence of the bubble shape in the final $\mathrm{S} / \mathrm{L}$ interface position.

3. Preheating phase duration. Analogously, this parameter modifies the shape of the liquid bubble generated during the Preheating phase and modifies the position of the $\mathrm{S} / \mathrm{L}$ interface and its velocity during the Final heating phase. The duration of this phase, originally of $480 \mathrm{~s}$ ( $8 \mathrm{~min}$ ), was adapted to the real flight execution, ranging from 7 to $11 \mathrm{~min}$.

A summary of the experiment parameter ranges explored during the campaign is given in Table 3.

\subsection{Experiment data}

The experiment was designed to collect and store the following timestamped outputs: four high-resolution set of images (two per Monitoring Station), temperature records at different relevant locations and gravity level measurements, provided by Novespace in a daily basis.

\subsubsection{Video acquisitions}

Two cameras were operated in parallel per Monitoring Station to observe the experiment evolution from two different views. The lateral camera, which focuses the mid section of the Experiment cell, was placed facing one of the lateral sides (see Fig. 4) to record the melting front progress. The top camera, on the other hand, observed the experiment from the $+\mathrm{Y}$ direction to provide information on the bidimensionality of the evolution, particularly, during the Final heating phase. Cropped images can be seen in Fig. 7.

As commented above, two different acquisitions rates were used depending on the experiment phase: $1 \mathrm{~Hz}$ for the Preheating and Cooling phases, and $10 \mathrm{~Hz}$ for the Final heating phase. These rates were a 
compromise solution between having enough (with more than comfortable margins) time resolution of the relevant phenomena, in accordance to physical timescales, and avoiding the buffer overload that can lead to the loss of the acquired frames.

\subsubsection{Temperature measurements}

The recording of temperatures was crucial for the later analysis of results and its comparison with the numerical model. Twelve J-thermocouples with operative range between -50 and $250^{\circ} \mathrm{C}$, were distributed in various locations. Eight of them were connected to an OMEGA USB Acquisition Module providing a resolution of $0.2 \mathrm{C}$ and an uncalibrated accuracy of $1^{\circ} \mathrm{C}$. The remaining four were fed to the Arduino making the thermal control of the peltiers via high precision thermocouple amplifiers.

Their readings, which were stored from the very beginning of each flight, were classified from an operational point of view in two sets: Experimental and Control temperatures.

Experimental temperatures.

- Four thermocouples (two per Monitoring Station; one per heat exchanger) were installed inside a drill performed up to the mid section of the heat exchanger, as close as possible to the Experiment cell, to monitor the effective cell temperature at $1 \mathrm{~Hz}$.

- Four thermocouples were located inside the Zarges Box to record the environment temperature at $1 \mathrm{~Hz}$. This temperature suffered oscillations with the opening and closing of the Zarges box for the installation and removal of cells.

- One thermometer monitored continuously the aircraft air cabin temperature. This temperature was required to be fairly below $28^{\circ} \mathrm{C}$, the n-octadecane melting point. A typical parabolic flight cabin temperature ranges from 21 to $23^{\circ} \mathrm{C}$ but to minimise the effect on the experiments, cabin temperature was maintained controlled to $21^{\circ} \mathrm{C}$ by the pilots with help of Novespace staff. Therefore, undesired melting of the PCM inside the Experiment cells when stowed was not observed.

Control temperatures. Four thermocouples were used to perform the peltier temperatures control, as depicted in Sec. 3.3.2. Their acquisition rate was $5 \mathrm{~Hz}$ to provide proper feedback to the control loop.

An example of the measured temperatures at Monitoring Station \#1 is illustrated in Fig. 9, where the experiment temperatures of peltiers \#1, 2 and the Zarges box are represented.

\subsubsection{Novespace gravity level measurements}

Novespace provided gravity level profiles during the flight and other complementary information, including the airplane attitude and flight level, recorded at $10 \mathrm{~Hz}$. Additionally, the full acceleration profile gives an overview of all the flight manoeuvres, information about the manoeuvres precision and the minimum microgravity level reached during the flight.

We find these measurements useful to identify any detrimental perturbation affecting the liquid behaviour. In fact, due to the imperfect gravity level in some parabolas, the contact line pinning was not effective and the liquid paraffin wetted completely the cell rails (see Fig. 12(a-d), for reference). Fortunately, this situation was only observed in some experiments throughout the full campaign.

Finally, a significant offset between Novespace readings and the experiment timestamp was detected. Comparing the images recorded with the acceleration profiles, we determine a difference of approximately $13 \mathrm{~s}$. We note that a more accurate estimation of this offset is not required, provided the experiment timescales.

\subsubsection{Scientific output}

To sum up, the science data gathered consists on a compilation of:

- Four timestamped videos capturing the evolution of the S/L front.

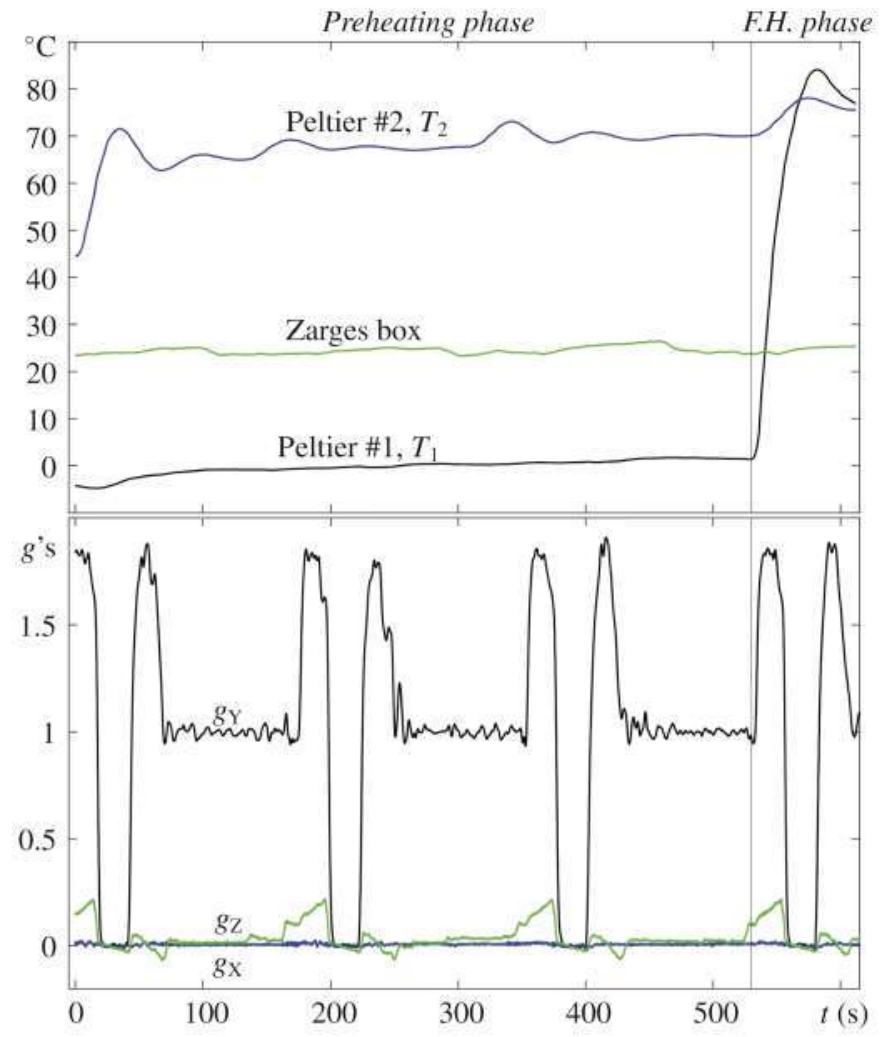

Fig. 9. Example of temperature (upper) and gravity level (lower, reproduced from Novespace) measurements recorded during the TEPiM experiment. Each profile is labelled and the transition from the Preheating phase to the Final heating phase highlighted with a vertical line. (For interpretation of the references to colour in this figure legend, the reader is referred to the Web version of this article.)

Two per Monitoring Station, observing the Experiment cell from along $\mathrm{Z}$ (lateral view) and $\mathrm{Y}$ (top view) axes.

- Timestamped temperature records of the $-\mathrm{X}$ face of the Experiment cells, and ambient temperature.

- Timestamped gravity level measurements, provided by Novespace.

- Timestamped logs of the beginning and end of the microgravity phases, for temporal reference only.

\section{Preliminary results and lessons learned}

Although not all of the 54 runs executed provided the expected results, the TEPiM experiment was successful in many ways. A systematic analysis of experiments will be presented separately, including, among other things, additional simulations and the validation of the numerical model. The main preliminary results and lessons learned are summarised hereafter.

\subsection{Effect of Marangoni convection: comparison of Marangoni and Reference cells}

Fig. 10 shows two images taken at the end of the microgravity phase from (a) Marangoni and (b) Reference cell experiments in $20 \mathrm{~mm}$ PCM height geometries. The effect of Marangoni convection can be clearly observed in the region near the liquid-air interface, where a greater advance of the $S / L$ front is achieved.

Besides this qualitative analysis, we have processed the corresponding videos and measured the apparent liquid area at the lateral wall $A_{L}$ (marked with red lines). The time evolution of the liquid fraction in both Experiment cells is shown in panel (c). During the Preheating phase, which lasted around $550 \mathrm{~s}$, the Marangoni cell 


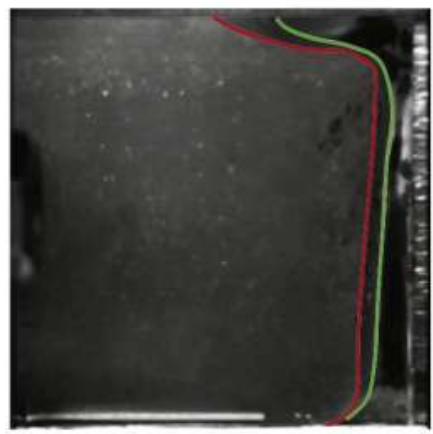

(a) Marangoni cell

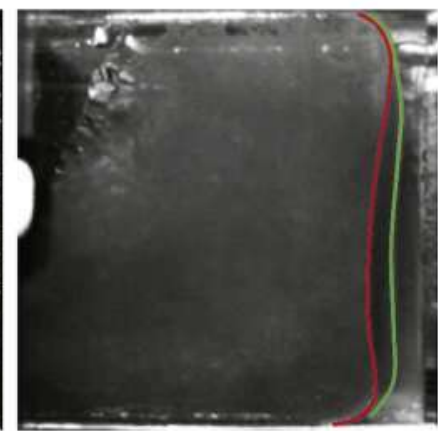

(b) Reference cell

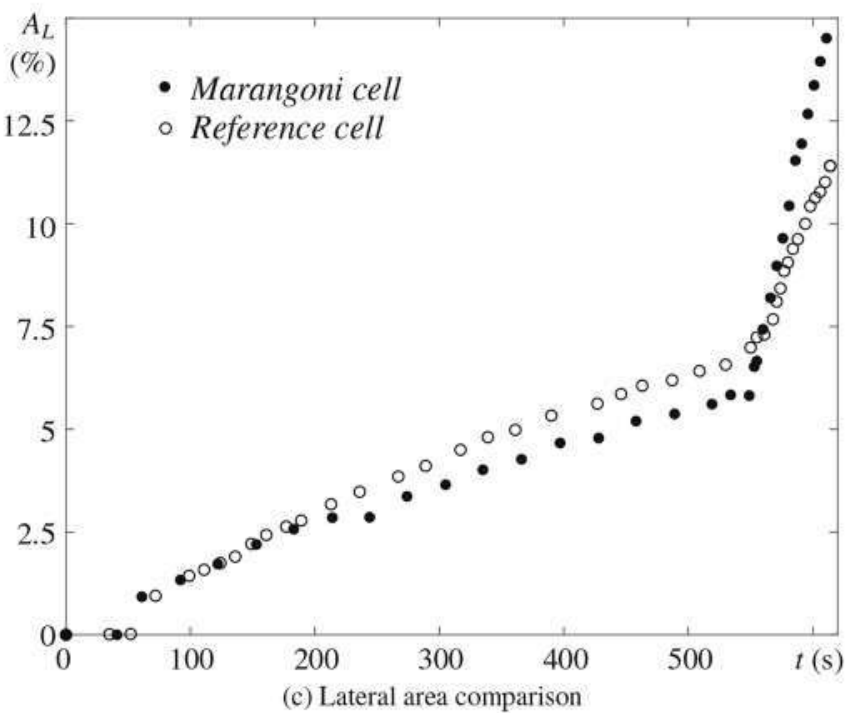

Fig. 10. Comparison of Marangoni and Reference cell experiments. (a, b) Final snapshots of (a) Marangoni cell and (b) Reference cell, showing the final S/L front. The lateral and central solidification fronts are highlighted with red and green lines, respectively. (c) Measurement of the liquid area in the lateral PMMA wall during analogous experiments. During the Final heating phase of these experiments, the liquid area in the Reference cell increases a $64 \%$ with respect to their initial value, while thermocapillary effects enhance substantially the melting rate in the Marangoni cell increasing the liquid area approximately a $150 \%$. (For interpretation of the references to colour in this figure legend, the reader is referred to the Web version of this article.)

experiment reached a liquid fraction of $\sim 6 \%$, while the Reference cell achieved roughly a $7 \%$ of liquid, for similar temperature profiles. This provides an approximate melting rate of $0.011-0.012 \% / \mathrm{s}$.

During the Final heating phase, the effect of increasing temperatures can be easily measured in the Reference cell, providing a final liquid fraction of $11.5 \%$. This yields a $64 \%$ increase with respect to the end of the Preheating phase, and an associated melting rate of $0.075 \% / \mathrm{s}$. In the Marangoni cell experiment, on the other hand, the final liquid fraction is approximately a $15 \%$. We observe almost a $150 \%$ increase in the melted region, with a melting rate of $0.15 \% / \mathrm{s}$. Since the substantial difference between Reference and Marangoni cell experiments is the presence of an air-liquid interface, one may conclude that, for this particular run, the Marangoni effect has promoted the heat transfer by a factor of 2 .

We note that a similar behaviour is expected in the central plane of the cell, the region of interest for the TEPiM experiment. As suggested by the central S/L front locations highlighted in the pictures, they seem to follow a similar trend to the lateral evolution but scaled by a certain factor. We find it reasonable to consider this factor approximately equal in both Marangoni and Reference cells, and thus, this effect is filtered somewhat when the relative evolution is analysed.

This result supports the use of Marangoni convection as a heat transfer enhancer and suggests the improvement of the PCMs performance for thermal control in the weightless condition of space missions. The final processing of all experiment runs, which include different geometries, is ongoing and associated results will be presented separately in the near future.

\subsection{Effect of PCM porosity during melting}

In general, the porosity of PCMs and its detrimental effect for thermal control is relatively well known, due to the effective reduction of the solid conductivity. In addition, the associated air retained is ejected to the liquid phase as small bubbles during melting. With gravity, the ejected bubbles are pushed upwards and their presence is concentrated on the upper boundary of the system. Therefore, the thermal behaviour is affected just locally.

The experimental procedure followed here, on the other hand, drives the formation of a liquid phase surrounded by the solid PCM (see Sec. 4, for details). During this process, some bubbles arose from the solid and concentrated in the upper boundary of the liquid phase near the aluminium wall. They sometimes coalesce to create a big air bubble that modifies locally the properties of the system, both thermally and optically. These bubbles, however, were eventually pushed away if the liquid-air interface creation occurred seconds prior the microgravity phase. Otherwise, they remained in the liquid and followed the flow streamlines, potentially modifying the experiment evolution. We illustrate a bubble creation process in microgravity in Fig. 11, until its later ejection during the hypergravity phase following the parabola. Due to the uncertainties in the thermal behaviour of these processes, we have put aside the affected runs for the time being.

This result suggests itself the need of reducing the PCM porosity. This may be achieved by improving the cells filling procedure, using, for example, vacuum during the process; or a controlled solidification that reduces the quantity of air retained in the solid phase. We note that

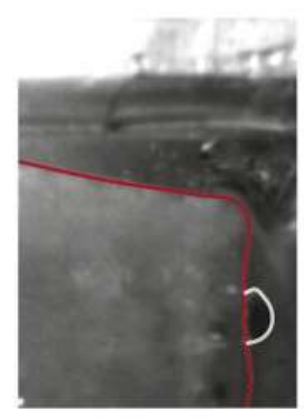

(a) $t=0.000 \mathrm{~s}$

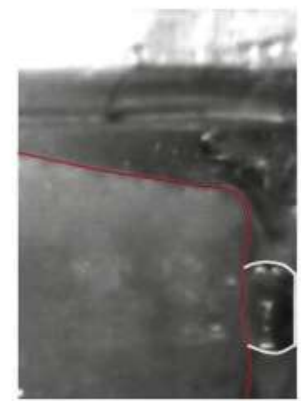

(d) $t=10.201 \mathrm{~s}$

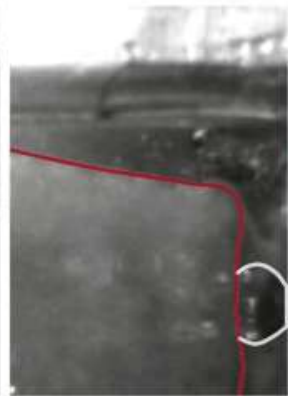

(b) $t=0.092 \mathrm{~s}$

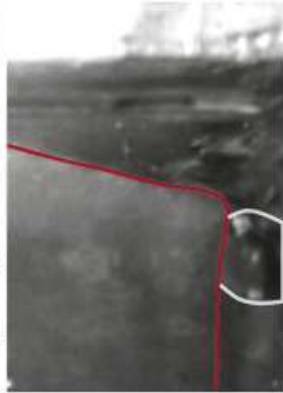

(e) $t=11.482 \mathrm{~s}$

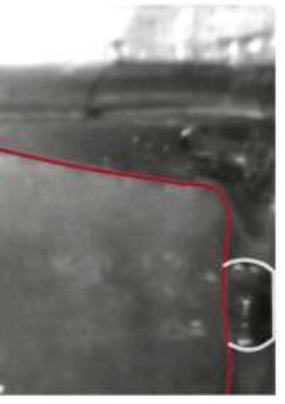

(c) $t=0.183 \mathrm{~s}$

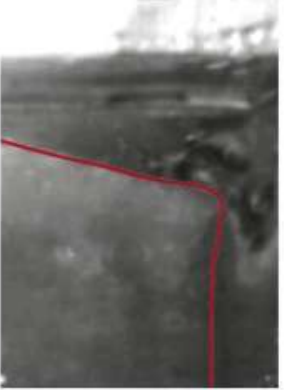

(f) $t=11.710 \mathrm{~s}$
Fig. 11. Snapshots showing the time evolution of a bubble creation in microgravity (a-d) and its ejection during the parabola transition to hypergravity (e-f). The steady location observed in microgravity perturbs locally the thermal behaviour of the experiment. For visual reference, the lateral S/L front (red line) and the bubble (white line) are highlighted. Times referred to panel (a) are labelled in each snapshot. (For interpretation of the references to colour in this figure legend, the reader is referred to the Web version of this article.) 


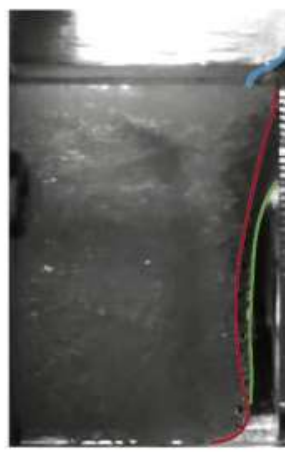

(a) $\sim 1 g_{0}$

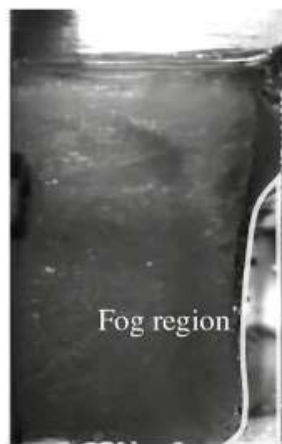

(d) $\sim 0.05 g_{0}$

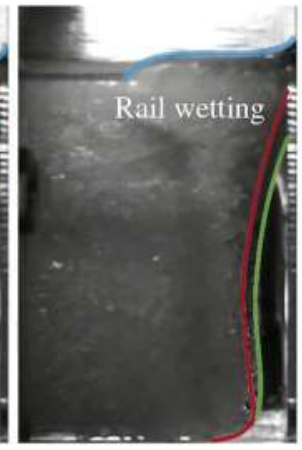

(b) $1.4 g_{0}$

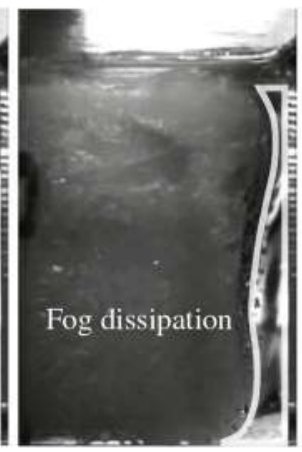

(e) $0.05 \rightarrow 1.8 g_{0}$

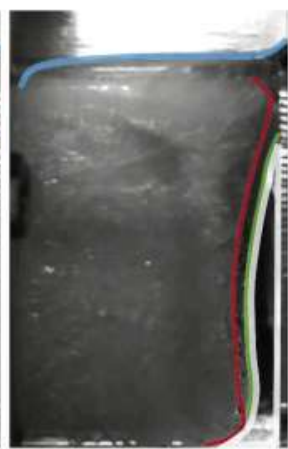

(c) $\sim 1.8 g_{0}$

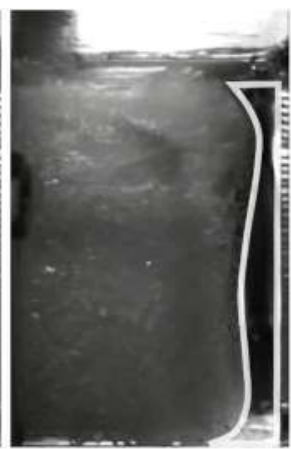

(f) $1.8 g_{0}$
Fig. 12. Snapshots showing six different lapses of the Final heating phase of a Marangoni cell experiment: $(\mathrm{a}-\mathrm{c})$ time evolution of the rail wetting in hypergravity during the pull-up phase, (d) fog region observed in microgravity, (e) fog dissipation during the transition to hypergravity of the relevant parabola, (f) end of the Final heating phase. The lateral and central S/L front locations and the liquid location in the rail are highlighted by red, green and light blue lines, respectively. The optical properties modification in delimited areas is evident, suggesting the existence of microbubbles solved in the fluid that remain distributed in the liquid during microgravity, altering its optical behaviour. (For interpretation of the references to colour in this figure legend, the reader is referred to the Web version of this article.)

the cells were filled by liquid PCM that solidified naturally at the laboratory temperature, in a passive and not controlled manner.

\subsection{Contact line pinning and microgravity disturbances of the parabolic flight}

Due to the imperfect microgravity level during some parabolas, the (uncertain) forcing threshold for the liquid phase to loss the contact line pinning and wet completely the cell rail was surpassed in some runs. In these cases, the development of Marangoni convection was not properly achieved.

In Fig. 12(a-c), the wetting process can be observed, where the liquid (marked in light blue) gradually occupies the rail. The uncontrolled liquid-air interface during the microgravity portion of the experiment diminished somewhat the associated thermocapillary effect. This effect is captured in panel (f), which can be compared to the final S/L front shown in Fig. 10 (a).

Fortunately, this undesired liquid displacement occurred in just some runs throughout the experimental campaign. This demonstrates the excellent performance of cell rails and coating to pin the contact line.

\subsection{Visualization issues during the flight campaign}

The careful revision of experiments revealed the existence of an opaque fog in the liquid phase evident during the microgravity phase. It appeared in the liquid paraffin preventing the $\mathrm{S} / \mathrm{L}$ interface visualization.

An example of this phenomenon is shown in Fig. 12(c-f), where the affected area is highlighted. Snapshots correspond to the Final heating phase of a Marangoni cell experiment. The optical behaviour of the liquid suffers a modification in the absence of gravity [panels (d, e)], where the liquid is captured in a lighter grayscale, compared to snapshots in gravity and hypergravity [panels (a-c,f)], where a dark grayscale is observed. This suggests the presence of microbubbles suspended in the fluid that altered optical paths and, therefore, prevented the consistent visualization of the central S/L front.

As discussed above, PCM porosity favours the presence of bubbles in the liquid phase. After the first flight completion, the team decided to subject the PCM to a slower (but still natural) cooling process, with no active controlled temperature. This attempt was unsuccessful and the same type of undesired effects were encountered in subsequent flights.

We note that, despite this obstacle, it has been possible to process the relevant information during the microgravity phase of many experiments (as shown in Fig. 10).

\subsection{Performance of the Active Thermal Control}

In light of the long phenomena timescales involved in PCMs melting and the relatively short microgravity phase duration, great effort was applied to design a high performance Thermal Control System.

In this sense, (quasi)steady temperature differences of $\sim 70^{\circ} \mathrm{C}$ were achieved between peltiers. Also, the system was capable to nullify these offsets in a very short time ( $\sim 30 \mathrm{~s})$ and to maintain high temperatures $\left(\sim 80^{\circ} \mathrm{C}\right)$ in both devices. This performance was mandatory to achieve a fast melt of the solid paraffin bridge, which confines the liquid during the Preheating phase, in order to start the microgravity phase with enough fluid present to observe Marangoni convection.

\section{Conclusions}

In this paper, we describe the scientific objectives, design, preparation and performance of the TEPiM experiment, which investigates the enhancement of heat transfer in PCMs in a weightless environment by fostering the development of Marangoni convection.

PCMs offer promising applications for thermal control in space, but have the drawback of its reduced heat transfer (compared to that under normal gravity conditions) due to the lack of convection in the liquid phase. There are relatively few microgravity experiments analysing the particularities of these devices in weightlessness, and none of them that we are aware of have focused on thermocapillary effects. Furthermore, the real execution of experiments has revealed issues not identified in (ground) preparatory phases, providing important insight on the challenges of developing future microgravity experiments of this nature.

From a scientific point of view, the TEPiM experiment has demonstrated the feasibility of exploiting the Marangoni convective flow for developing more efficient space thermal control devices. We expect that the future analysis of the experimental data will provide further understanding of thermoregulation with PCMs in microgravity.

The main obtained conclusions are:

(i) Preliminary results support that the Marangoni effect greatly stimulates heat transfer in PCMs under microgravity conditions.

(ii) Problems arising from the air retained inside the PCM suggest that further studies in this area are needed.

(iii) Numerical modelling of the heat transfer through the cell walls is necessary to reproduce real experiments.

(iv) Due to the different heat transfer properties of the PMMA and the air layer, a pair of extra thermocouples could have been useful to characterize the thermal configuration. In this sense, the enhancement of the "adiabatic" surfaces is also desirable for future designs. 
(v) For future experiments, alternative optical and illumination configurations should be investigated to ease the processing of the images.

\section{Acknowledgements}

This work was supported by the ESA Education Office in connection with the Fly Your Thesis! Programme and the 65th ESA Parabolic Flight Campaign, and by the E-USOC. We thank the European Low Gravity Research Association (ELGRA) for their valuable scientific support and Novespace for all their technical advice and contributions during the project. We thank the Escuela Técnica Superior de Ingeniería Aeronáutica y del Espacio at the Universidad Politécnica de Madrid, which have supported this project from the beginning and, in particular, the research group of Ciencias y Operaciones Aeroespaciales for their invaluable effort. Finally, we thank the Center for Computational Simulation (CCS) at the Universidad Politécnica de Madrid.

\section{References}

[1] S.A. Memon, Phase change materials integrated in building walls: a state of the art review, Renew. Sustain. Energy Rev. 31 (2014) 870-906 https://doi.org/10.1016/j. rser.2013.12.042

[2] T.Y. Kim, B.S. Hyun, J.J. Lee, J. Rhee, Numerical study of the spacecraft thermal control hardware combining solid-liquid phase change material and a heat pipe, Aerosp. Sci. Technol. 27 (2013) 10-16 https://doi.org/10.1016/j.ast.2012.05.007.

[3] D. Fernandes, F. Pitié, G. Cáceres, J. Baeyens, Thermal energy storage: "How previous findings determine current research priorities", Energy 39 (2012) 246-257 https://doi.org/10.1016/j.energy.2012.01.024.

[4] M. Giangi, F. Stella, E. Leonardi, G. De Vahl Davis, A numerical study of solidification in the presence of a free surface under microgravity conditions, Numer. Heat Transf. Part A Appl. 41 (2002) 579-595 hittps;//doi.org/10.1080/ 104077802317418223.

[5] W.R. Humphries, E.I. Griggs, A Design Handbook for Phase Change Thermal Control and Energy Storage Devices, Technical Report, 1977.

[6] T.D. Swanson, G.C. Birur, NASA thermal control technologies for robotic spacecraft, Appl. Therm. Eng. 23 (2003) 1055-1065 https://doi.org/10.1016/S1359-4311(03) 00036-X.

[7] G. Xiaohong, S. Xiange, Z. Miao, T. Dawei, Influence of void ratio on thermal stress of PCM canister for heat pipe receiver, Appl. Therm. Eng. 94 (2012) 615-621 https://doi.org/10.1016/j.applthermaleng.2015.10.003.

[8] D.V. Lyubimov, T.P. Lyubimova, A. Croell, P. Dold, K.W. Benz, B. Roux, Vibrationinduced convective flows, Microgravity Sci. Technol, 11 (1998) 101-106.

[9] P. Dold, K.W. Benz, A. Croll, B. Roux, D. Lyubimov, T. Lyubimova, R. Scuridyn, Vibration controlled convection - preparation and perspectives of the Maxus 4 experiment, Acta Astronaut. 48 (2001) 639-646 https://doi.org/10.1016/S00945765(01)00033-9.

[10] S.Z. Shuja, B.S. Yilbas, O. Momin, Laser repetitive pulse heating and melt pool formation at the surface, J. Mech. Sci. Technol. 25 (2011) 479-487 https://doi.org/ 10.1007/s12206-010-1208-4.

[11] C. Gau, R. Viskanta, Melting and solidification of a pure metal on a vertical wall, J. Heat Transf. 1 (1986) 17-30, https://doi.org/10.1115/1.3246884.

[12] Y. Wang, A. Amiri, K. Vafai, An experimental investigation of the melting process in a rectangular enclosure, Int. J. Heat Mass Transf. 42 (1999) 3659-3672 https://doi. org/10.1016/S0017-9310(99)00024-1.

[13] H. Shokouhmand, B. Kamkari, Experimental investigation on melting heat transfer characteristics of lauric acid in a rectangular thermal storage unit, Exp. Therm. Fluid Sci. 50 (2013) 201-212 https://doi.org/10.1016/j.expthermflusci.2013.06. 010 .

[14] V. Pletser, S. Rouquette, U. Friedrich, J.F. Clervoy, T. Gharib, F. Gai, C. Mora, The 1st European parabolic flight campaign with the airbus A310 ZERO-G, Microgravity Sci. Technol. 28 (2016) 587-601 https:/doi.org/10.1007/s12217-016-9515-8.

[15] E.M. Alawadhi, Thermal analysis of a building brick containing phase change material, Energy Build. 40 (2008) 351-357 https://doi.org/10.1016/j.enbuild.2007. 03.001.

[16] N.S. Dhaidan, J.M. Khodadadi, T.A. Al-Hattab, S.M. Al-Mashat, Experimental and numerical investigation of melting of NePCM inside an annular container under a constant heat flux including the effect of eccentricity, Int. J. Heat Mass Transf. 67 (2013) 455-468 htips://doi.org/10.1016/j.ijheatmasstransfer.2013.08.002

[17] W.M. Haynes, D.R. Lide, T.J. Bruno, CRC Handbook of Chemistry and Physics: A Ready-Reference Book of Chemical and Physical Data, 97th Edition, CRC Press, Boca Raton, Florida, 2016 (Print).

[18] Alfa Aesar Octadecane datasheet, https://www.alfa.com/es/msds/?language =EE\&. subformat $=$ CLP1 \&sku=31954, Accessed date: 24 April 2019.

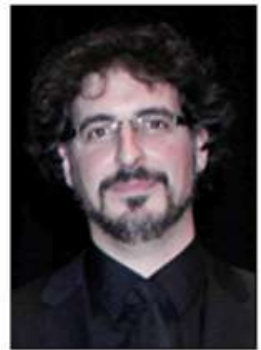

J.M. Ezquerro

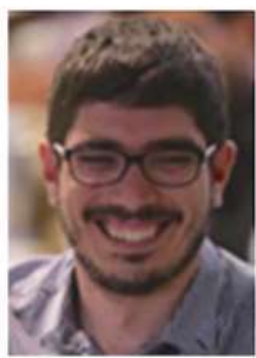

A. Bello

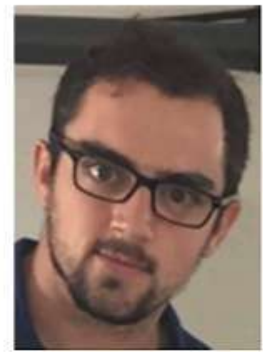

P. Salgado Sánchez

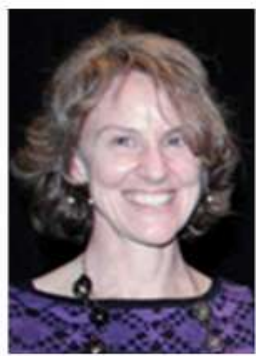

A. Laveron-Simavilla

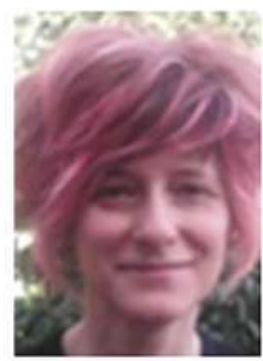

V. Lapuerta 